\title{
A rare case of ossification of ligamentum flavum presenting as dorsal myelopathy in achondroplasia
}

\begin{abstract}
Ossification of the ligamentum flavum is a spinal disorder that most commonly occurs in middle-aged men. It is most commonly prevalent in Japan and other East Asian countries such as Korea and China and usually presents with myelopathy. However, its occurence in achondroplasia is extremely rare. We present a rare case of spinal stenosis due to ossified ligamentum flavum in a 30 year old Indian female of achondroplasia presenting as dorsal myelopathy.
\end{abstract}

Keywords: ossification of ligamentum flavum, achondroplasia, myeloradiculpathy, thoracic spinal cord
Volume 7 Issue 5 - 2017

\author{
Tamajit Chakraborty, Divyam Sharma, Ashish \\ Goyal Madan VS \\ Department of Neurosurgery, Sir Ganga Ram Hospital, India
}

\author{
Correspondence: Divyam Sharma, Department of \\ Neurosurgery, Room No. F-34, Neurosurgery Office, Sir \\ Ganga Ram Hospital, Rajinder Nagar New Delhi, India,Tel \\ 91958225|293, Email divyam 17@gmail.com
}

Received: February 08, 2017| Published: October 12, 2017
Abbreviations: OLF, ossification of ligamentum flavum; OPLL, ossification of posterior longitudinal ligament; DISH, diffuse skeletal hyperostosis; PIVD, prolapsed inter-vertebral disc; CT, computed tomography; MRI, magnetic resonance imaging; HNP, herniated nucleus pulposus

\section{Introduction}

Ossification of the ligamentum flavum (OLF) was discovered by Polgar and further described by Yamaguchi and Isuruni. ${ }^{1}$ Among the thoracic myelopathies, its incidence is less than that of ossification of the posterior longitudinal ligament (OPLL) and higher than that of herniated nucleus pulposus (HNP). ${ }^{1}$ It is mostly prevalent in Japan and other East Asian countries such as Korea and China. ${ }^{2}$ Achondroplasia is a genetic disorder of bone growth with congenital spinal stenosis being a well known complication. ${ }^{3}$ Degenerative changes of the spine, such as disc herniation, degenerative spondylosis and arthrosis result in worsening of stenosis. ${ }^{4}$ However, symptomatic spinal stenosis due by ossification of the ligamentum flavum (OLF) in achondroplasia is extremely rare. ${ }^{5,6} \mathrm{We}$ present a rare case of spinal stenosis due to ossified ligamentum flavum in a 30 year old Indian female of achondroplasia presenting as dorsal myelopathy.

\section{Case presentation}

A 30-year-old Indian woman, non-smoker, known case of achondroplasia, presented with chief complaints of difficulty in walking along with flexor spasms since eight months along with bilateral progressive sensory loss in lower limbs for past four months. Neurological examination revealed power of $4 / 5$ in her left hip adductors and abductors and knee flexors, $3 / 5$ in knee extensors and $1 / 5$ in ankle dorsiflexors as well as planterflexors. Right side muscle group power was $5 / 5$; the left knee and ankle jerk were $3+$ and the right knee and jerk were $2+$. The patient had decreased touch, pain, temperature sensations in her leg and feet along with decreased vibratory sensations and proprioception in her both legs and feet with bilateral extensor plantars. Her perianal sensations and rectal tone were normal. CT and MRI dorsolumbar spine (Figure $1 \& 2$ ) revealed facet hypertrophy with OLF at D10-11 levels with cord compression and signal changes at D10-D12 level along with partial collapse of D11 vertebra and dural calcification at D11 and D12 levels (Figure $3 \& 4)$.
She underwent bilateral D10-11-12 pedicle screw and rod fixation and D10-D12 laminectomy with cord decompression. During surgery ossified ligamentum flavum was seen at D10-11 level which was firmly adhered to the dura with no discernible plane between OLF and the dura which was removed using a high-speed drill and curettes. Postoperatively, gradually the power and sensations improved and the flexor spasm were relieved, and the patient started walking with a slight limp of the left ankle.

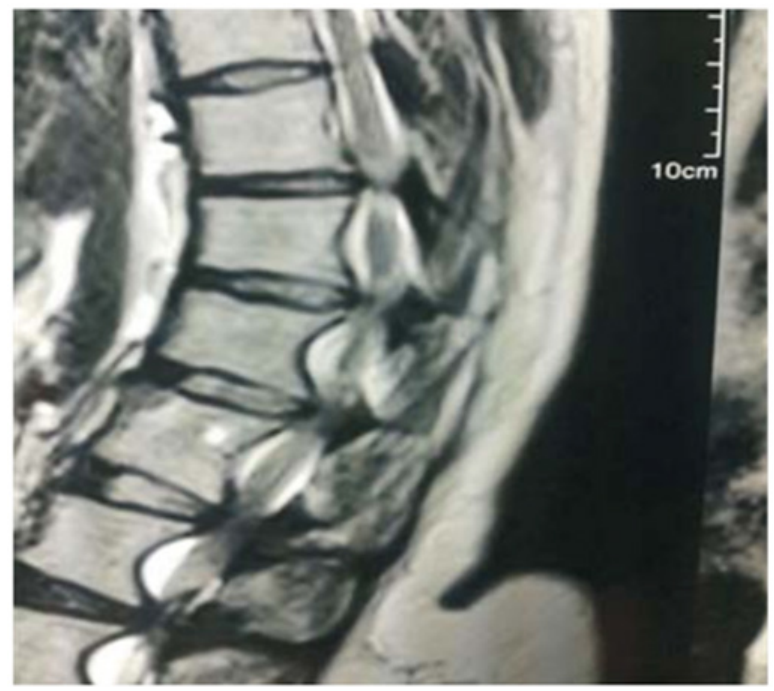

Figure I MRI Dorsolumbar Spine Sagittal View.

\section{Discussion}

Ossified ligamentum flavum (OLF) is a condition of heterotopic lamellar bone formation within the yellow ligament. ${ }^{2}$ Since the first description by Yamaguchi and Isuruni, thickened ligamentum flavum is being gradually appreciated as an important cause of thoracic myeloradiculopathy. ${ }^{7}$ OLF has been seen to be associated with trauma, diffuse idiopathic skeletal hyperostosis (DISH), ankylosing spondylosis, hemochromatosis, fluorosis and disorders of calcium and phosphorous, ${ }^{8}$ however the underlying etiology and pathogenesis of OLF are still unknown. ${ }^{9}$ Recent studies indicate that the development of OPLL is associated with certain genetic factors ${ }^{10,11}$ which may also play a specific role in the origin of OLF (12). The lower thoracic 
spine (T9-12) is the most frequently affected, while the mid-thoracic spine (T5-8) is rarely affected. ${ }^{13} \mathrm{CT}$ and MRI are the two most common diagnostic modalities with $\mathrm{CT}$ being the more sensitive tool for diagnosis. ${ }^{12}$ Surgery remains the treatment of choice and several posterior decompressive methods (open-door laminectomy, en-bloc laminectomy, fenestration, hemilaminectomy, and key-hole foraminotomy) for the ossified lesions are advocated. ${ }^{14}$ All treatment options should be weighed against each other in achondroplasia due to the congenital narrowing of the spinal canal and the thickened laminae and increased chances of perioperative complications such as a dural tear which are common during multilevel laminectomies. ${ }^{15}$ We did a laminectomy with decompression using high speed drill and curettes along with fusion without any intraoperative complications and achieved significant gradual clinical improvement.

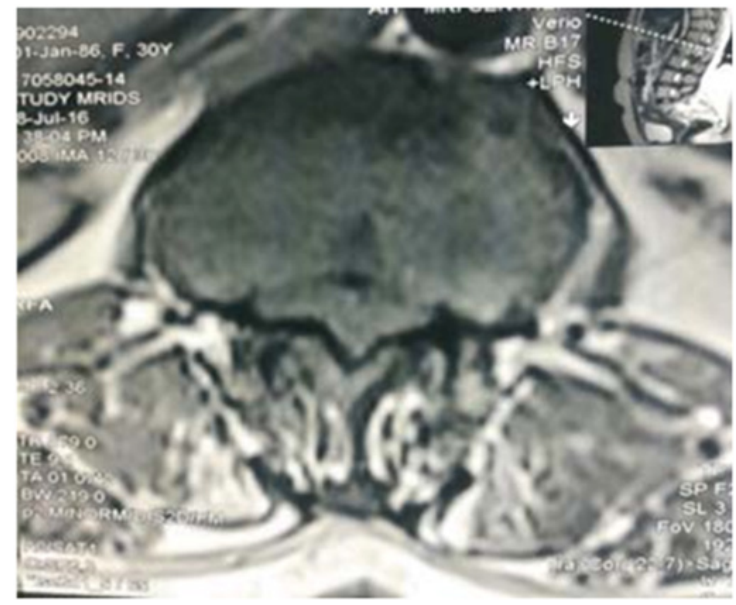

Figure 2 MRI Axial Section.

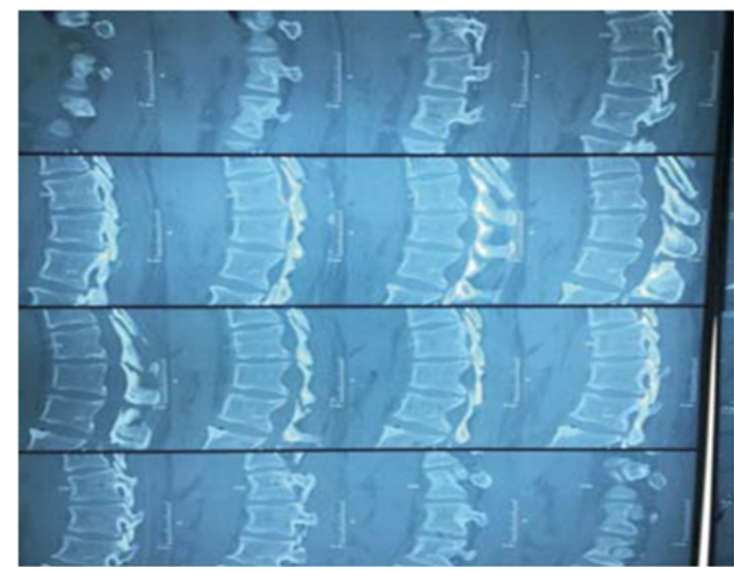

Figure $3 \mathrm{CT}$ scan of the dorsolumbarspine sagittal view.

\section{Acknowledgements}

The authors would like to acknowledge the patient who agreed to be a part of this case study. Also we would like to acknowledge our hospital's academic department and library which helped us immensely with the research work.

\section{Conflicts of interest}

None.

\section{Funding}

None.

\section{References}

1. Fong SY,Wong HK. Thoracic myelopathy secondary toligamentumflavum ossification. Ann Acad Med Singapore. 2004;33(3):340-346.

2. Miyasaka K, Kaneda K, Sato S. Myelopathy due to ossification or calcification of the ligamentumflavum: Radiologic and histologic evaluations. AJNR Am J Neuroradiol. 1983;4(3):629-632.

3. Hunter AG, Bankier A, Rogers JG, et al. Medical complications of achondroplasia: a multicentre patient review. $J$ Med Genet. 1998;35(9):705-712.

4. Lutter LD, Langer LO. Neurological symptoms in achondroplasticdwarfs: surgical treatment. J Bone Joint Surg Am. 1977;59(1):87-92.

5. Suzuki K1, Kanamori M, Nobukiyo M. Ossification of the thoracic ligamentumflavum in an achondroplastic patient: a case report. J Orthop Surg (Hong Kong). 2008;16(3):392-395.

6. Saito K, Miyakoshi N, Hongo M. Congenital lumbar spinal stenosis with ossification of the ligamentumflavum in achondroplasia: a case report. Journal of Medical Case Reports. 2014;8:88.

7. Miyasaka K, Kiyoshi K, Ito T. Ossification of spinalligaments causing thoracic radiculomyelopathy. Radiology. 1982;143(2):463-468.

8. Resnick D, NiwayamaG. Entheses and entheropathyanatomic pathologic and radiologic correlation. Radiology. 1983;146(1):1-9.

9. Li KK, Chung OM, Chang YP, et al. Myelopathy causedby ossification of ligamentumflavum. Spine. 2002; 27(12):E308-E312.

10. Koga H, Sakou T, Taketomi E, et al. Genetic mapping of ossification of the posterior longitudinal ligament of the spine. Am J Hum Genet. 1998;62(6):1460-1467.

11. Maeda S, Koga H, Matsunaga S, et al. Gender-specific haplotype association of collagen alpha2 (XI) gene in ossification of the posterior longitudinal ligament of the spine. J Hum Genet. 2001;46(1):1-4.

12. Aizawa T, Sato T, Sasaki H, et al. Thoracic myelopathy caused by ossification of the ligamentumflavum: clinical features and surgical results in the Japanese population. J Neurosurg Spine. 2006;5(6):514-519.

13. Yoon SH, Kim WH, Chung SB, et al. Clinical analysis of thoracic ossified ligamentumflavum without ventral compressive lesion. Eur Spine J. 2011;20(2):216-223

14. N Lang, H S Yuan, H L Wang. Epidemiological survey of ossification of the ligamentumflavum in thoracic spine: CTimaging observation of 993 cases, Eur Spine J . 2013;22(4):857-862.

15. Shiokawa K, Hanakita J, Suwa H, et al. Clinical analysis and prognostic study of ossifiedligamentumflavum of the thoracic spine. J Neurosurg. 2001;94(suppl 2):221-226. 